Utopie de la communication interne : vers une « maïeutique managériale de la confiance » dans l'organisation vertueuse

\title{
Frédéric Ely
}

\section{QpenEdition}

\section{Journals}

Édition électronique

URL : http://journals.openedition.org/communicationorganisation/4958

DOI : 10.4000/communicationorganisation.4958

ISSN : 1775-3546

Éditeur

Presses universitaires de Bordeaux

Édition imprimée

Date de publication : 1 juin 2015

Pagination : 197-216

ISSN : 1168-5549

\section{Référence électronique}

Frédéric Ely, « Utopie de la communication interne : vers une « maïeutique managériale de la confiance » dans l'organisation vertueuse »,Communication et organisation [En ligne], 47 | 2015, mis en ligne le 01 juin 2018, consulté le 19 avril 2019. URL : http://journals.openedition.org/ communicationorganisation/4958 ; DOI : 10.4000/communicationorganisation.4958 


\title{
Utopie de la communication interne : vers une " maïeutique managériale de la confiance» dans l'organisation vertueuse
}

\author{
Frédéric Elỵ' \\ "Eh bien, n'as-tu pas entendu dire que je suis le fils d'une \\ sage-femme, et que j'ai l'art d'accoucher les esprits, comme elle \\ avait celui d'accoucher les femmes?" \\ Platon, Théétète
}

Depuis plusieurs décennies, la confiance, qu'elle soit interpersonnelle, organisationnelle ou inter-organisationnelle, a fait l'objet de très nombreuses et grandissantes recherches, tant dans les sciences économiques, de gestion, que sociales ou de l'information et de la communication. Son importance pour la performance de l'organisation n'est plus à démontrer, tout autant que ses rapports étroits avec les domaines du management et de la communication.

Alors que la santé mentale au travail est devenue un enjeu majeur de santé publique, nous constatons que, de nos jours encore, dans bien des organisations, les conditions sont loin d'être réunies pour favoriser, au-delà des récurrentes bonnes intentions, cette confiance entre les collaborateurs. Devant ce que nous appelons une « désertification de l'humain dans l'organisation » (Ely 2011), ajoutée à notre diagnostic de « maladie de la communication interne » (Ibid.), face à un contexte de malaise social et de crise managériale, nous proposons de construire notre problématique dans le prolongement des travaux de Breton (1997), autour des manifestations d'une communication interne devenue utopique. L'objectif de cette recherche exploratoire est ainsi de questionner le concept de confiance devant les menaces socioprofessionnelles en présence, mais aussi devant les opportunités que peut offrir un rapprochement original entre le monde du management et celui de la philosophie, via les apports des

1 Maître de Conférences en SIC, Université de Nice Sophia Antipolis, UFR Lettres, Art et Sciences Humaines, Département Sciences de la Communication. Laboratoire I3M ; http://i3m.univ-tln.fr/ELY-Frederic.html ; frederic.ely@unice.fr 
vertus morales et de la maïeutique socratique : en quoi ces derniers peuvent-ils constituer une ressource intéressante pour le management des communautés ?

Pour cadrer notre réflexion, compte tenu de notre champ de recherche interdisciplinaire, centré sur les rapports complexes entre communication des organisations et management, nous avons choisi de positionner notre étude dans le cadre de la confiance organisationnelle comme, " au sens large, la confiance au sein de l'organisation et, au sens strict, la confiance que les salariés peuvent placer dans les dirigeants de leur entreprise " (Neveu 2004 : 1071). Nous retenons également une définition complémentaire de la confiance comme " relation qui lie le salarié à son supérieur et à son employeur [...] un état psychologique comprenant l'acceptation d'une vulnérabilité fondée sur des croyances concernant les intentions ou le comportement d'une autre personne ». (Ibid. : 1080). Nous choisissons par ailleurs de nous référer à une définition de la vertu, dans son acception morale, comme "puissance d'bumanité "(Conte-Sponville 1995 : 9), ainsi qu'à une définition normative de la communication interne en tant qu' « outil du management » (Mucchielli 2001) et telle qu' « un ensemble de principes d'actions et de pratiques visant à donner du sens et favoriser l'appropriation, à donner de l'âme pour favoriser la cohésion et inciter chacun à mieux communiquer pour favoriser le travail en commun » (Détrie \& Broyez 2001). Au plan méthodologique, nous adoptons un positionnement épistémologique constructiviste dit « expérientiel » (Mucchielli 2004 : 17), en recherchant les meilleures inspirations interactionnistes de l'école de $\mathrm{Palo} \mathrm{Alto}^{2}$, dans un système d'interdépendances entre les domaines de la communication interne, des ressources humaines et du management. En complément des résultats qualitatifs et quantitatifs obtenus lors d'un travail de thèse centré sur un objet encore peu exploré (Ely 2007), nous proposons de conduire notre réflexion par une observation empirique de notre terrain : un dispositif numérique de collaboration à distance et de communication interne que nous avons piloté, durant trois années successives, au sein d'une grande entreprise française d'assurance.

Dans un premier temps, nous poserons les bases d'une utopie de la communication interne en nous appuyant sur un rappel des principaux facteurs de détérioration de la confiance organisationnelle. Dans un second temps, nous questionnerons la restauration possible de cette dernière au regard d'une certaine éthique managériale par les vertus morales. Enfin, dans un troisième temps, en examinant nos pratiques de Community Manager, nous proposerons d'approcher ce que nous nous sommes efforcé d'appliquer sur notre terrain expérimental et que nous désignons par les termes de maïeutique managériale de la confiance.

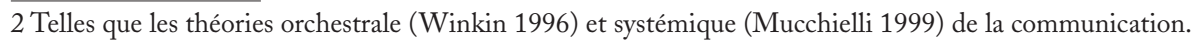




\section{Une confiance organisationnelle détériorée : base d'une utopie de la communication interne?}

Le système professionnel global français que nous observons de longue date, présente de multiples conditions susceptibles de détériorer la confiance des acteurs de l'organisation.

Nous faisions déjà état, dans nos précédents travaux, à l'appui des recherches de nombreux auteurs ${ }^{3}$, d'un contexte organisationnel pathogène, au point de conclure au diagnostic d'une " maladie de la communication interne » de l'organisation (Ely 2010 : 259), après avoir distingué tout un nœud de difficultés, de paradoxes organisationnels et leur nocivité dans les domaines de l'organisation du travail (rationalisation organisationnelle, RTT) des ressources humaines (souffrance au travail), du management (style managérial) et de la culture d'entreprise.

Devant ce contexte de darwinisme organisationnel, si les salariés-récepteurs, dans leur processus subi de "démentalisation »(Hirigoyen 2003), ne peuvent plus se trouver suffisamment réceptifs aux messages managériaux de communication interne, nous trouvons intéressant de questionner le concept de confiance au regard de son opposé, la défiance, dans l'organisation.

\section{Une défiance contre-productive pour l'organisation}

Devant un discours managérial idéaliste sur le travail prétendument libérateur, les nouvelles formes organisationnelles, dans les conditions d'un management perverti, tendent à conduire à une situation fort dépréciée. Aggravées par la crise économique, les distorsions entre les discours officiels et les réalités du travail sont légion : «les discours s'ornent de valeurs morales sans que les comportements en soient réellement imprégnés. Le changement loué dans les mots ne se retrouve pas dans les actions des dirigeants au grand désarroi des salariés. Ces derniers perdent confiance en leur direction. » (Batazzi 2005 : 147). Dès lors, l'insatisfaction des salariés se dégrade en défiance générale vis-à-vis de l'entreprise, situation de fait apparaissant comme une « crise durable, à combustion lente mais ravageuse " (Auriac \& Chanon $2012: 47$ ).

Un management pathogène, autoritaire, rationaliste, et une politique $\mathrm{RH}$, de ce fait, inattentive aux efforts des salariés, génère un manque de considération, de confiance envers le personnel qui suscite, en retour, cette forme de défiance envers l'organisation. Nous avions constaté ce phénomène de défiance mutuelle au sein de l'organisation observée (Ely 2011). Dans le contexte d'une grande structure fortement hiérarchisée, nous avions identifié la prégnance d'une culture assurancielle modelée par certaines attitudes

3 Dont les premiers : Dejours (1998, 2000) ; Dejours \& Bègue (2009) ; Dieguez (2009); Pezé (2010); Hirigoyen (2003) ; Méda (1996) ; Crozier \& Friedberg (1977) ; Des Isnard \& Zuber (2008) ; Hopquin (2008) ; Duterme (2002) ; Dufour (2003) ; Haddad (2013) ; Clot (2010) ; Gorz (1998) ; Rifkin (1995) ; Salengro (2006) ; Mintzberg (2004) ; Lojkine \&Maletras (2003) ; Peter \& Hull (1970) ; Ellul (1977). 
systématiquement suspicieuses et protectionnistes de dirigeants bien souvent ex-gestionnaires du risque d'assurance. Comme par une forme de déformation professionnelle, cette culture nous est apparue susceptible de contribuer à ce que les salariés soient davantage appréhendés par le système managérial sous cet angle restreint, abusif et paranoïaque du risque, plutôt que sous l'angle, plus ouvert, des opportunités en termes de ressources humaines...

Par ailleurs, au-delà des divers vecteurs de détérioration de la confiance évoqués plus haut, le spectacle politico-médiatico-financier, sans cesse réitéré, des affaires de certains hommes politiques ou chefs d'entreprises, les licenciements massifs, associés à des délocalisations abusives ou à certaines rémunérations disproportionnées aux sommets des pyramides, mettent en cause certaines pratiques managériales et donnent un sentiment d'indignation conduisant à la diffusion et à l'entretien d'une véritable défiance de la part des salariés vis-à-vis de l'entreprise, comme " rupture du lien entreprise-salarié » (TNS Sofres 2009 : 5), dans le cadre plus général d'une « société de défiance » ou d'une « fabrique de la défiance » (Algan et al., 2007, 2012)...

Cette défiance, contre-productive pour l'organisation, favorise des attitudes de repli sur soi, et d'individualisme, des comportements exclusivement orientés par la préservation des avantages individuels, au mépris de l'intérêt collectif. Un certain scepticisme quant à la légitimité des instructions hiérarchiques, l'altération de l'esprit d'initiative, et une adhésion dégradée à la culture de l'organisation, sont autant de comportements de nature à réduire la compétitivité de l'organisation et son acclimatation à des modèles plus vertueux tels que l'entreprise apprenante, démocratique, intelligente.

\section{Une utopie de la communication interne?}

Le chapitre qui précède nous invite à proposer de dégager une première conclusion relative aux effets pathogènes, en cascade, occasionnés sur la communication interne de l'organisation, par les différents vecteurs de détérioration de la confiance des acteurs de l'organisation. Ces derniers participent, selon nous, de l'entretien, au sein de l'organisation, d'une forme de syndrome, complexe et diffus, fait d'absurde, de non-sens et d' « incommunication » (Huisman 1985) interne, qui nous invite à proposer d'avancer les bases d'une utopie de la communication interne de l'organisation, dans la continuité des travaux de Breton (1997). Ce constat se trouve, à nos yeux, d'autant plus problématique que nous avons retenu, dans notre introduction, la communication interne comme «outil du management». Pour illustrer sommairement les termes de ce rapprochement, nous nous appuyons, de façon générale, et comme le fait l'auteur, sur la pensée et l'œuvre de Wiener, père de la cybernétique et d'une certaine " utopie wienérienne " (Breton 1997 : 11). Si cette utopie de la communication est bien décrite par l'auteur sur un plan sociétal, dans un contexte de "société de communication » et de « transparence sociale » (Breton 1997 : 59), en quoi pouvons-nous avancer 
sa transposition au plan plus particulier de la communication interne de l'organisation?

Nous appuierons notre proposition sur trois éléments fondamentaux que nous considérons comme dénominateurs communs en jeu : l'entropie sociale, la barbarie moderne et les machines à communiquer.

Utopie de la communication interne et " entropie sociale "

La communication, au sens général du terme, est caractérisée comme utopique dès lors qu'elle entre en jeu comme une forme de moyen providentiel de lutte contre une certaine "entropie sociale " (Breton 1997 : 33). Apparaît ainsi la notion de responsabilité de l'homme vis-à-vis de la société et de la nature dans laquelle "il vit pour "faire reculer localement l'entropie" " (Breton 1997 : 34), cet "Homme nouveau ", cet "Homo communicans [...] qui vit dans une société sans secret, un être tout entier tourné vers le social, qui n'existe qu'à travers l'information et l'échange, dans une société rendue transparente grâce aux nouvelles "machines à communiquer" »(Breton 1997 : 50). Si la communication et l'Homme apparaissent ici comme une panacée pour lutter contre l'entropie sociale, au plan général de la société, ne rencontrons-nous pas une utopie similaire dans le monde désenchanté de l'organisation vue sous un angle critique ? Cette organisation, ne fixe-t-elle pas, en effet, au Directeur de la communication interne, l'objectif - du reste, inatteignable - de lutter contre l'entropie de l'information et du sens, vers plus de cohésion interne ? La société de communication et la communication interne de l'organisation ne sont-elles pas exposées, l'une et l'autre, à des dérives similaires - et peutêtre contigües -, faites de "tautisme " (Sfez 1988), de communication pour la communication, de confusion entre l'information et le sens, d' " illusion de la libération par la communication »(Breton 1997 : 157), en ramenant ainsi l'organisation à l'échelle d'une socialité vide, celle d'une « société fortement communicante mais faiblement rencontrante » (Breton 1997 : 160) ?

Utopie de la communication interne et " barbarie moderne "

Le contexte de "barbarie moderne " (guerres, massacres collectifs du vingtième siècle, génocides, nazisme...) apparaît comme une autre condition constitutive de l'utopie de la communication (Breton 1997 : 75). Ne sommesnous pas, dans une certaine mesure, devant les dérives du travail rappelées cidessus et rencontrées, par exemple, au sein de France Télécom et ses séries de suicides sur le lieu de travail, devant une autre forme de «barbarie moderne »? Celle-ci n'a-t-elle pas ceci de particulier qu'elle est perçue par ceux-là même qui la mettent en œuvre (nous pensons au système de management et de communication interne inefficacement déployés avant et après les drames répétés), comme « un moyen destiné à mettre en œuvre un changement vers une société meilleure, "purifiée" »(Breton 1997 : 77) ? 
Utopie de la communication interne et "machines à communiquer"

Lordinateur est considéré comme le " cheval de Troie de l'utopie de la communication " (Breton 1997 : 107). Cette "machine à communiquer " (Breton 1997 :58) n'est-elle pas également le «cheval de Troie» d'une utopie de la communication interne, depuis ces dernières années, avec la mutation observée de ce domaine vers le numérique (Ely 2005a) ? La "révolution pacifique » (Champeaux \& Bret 2000) des TIC dans l'organisation, depuis la fin des années 1990, a en effet permis une révolution des usages méthodologiques et pratiques de la communication interne au même titre que : " nous assistons aujourd'hui au renouveau massif du discours utopique wienérien à travers le thème des autoroutes de données et de la "nouvelle frontière électronique"» (Breton 1997 : 19). De même, cette utopie de la communication interne dont nous proposons le constat, ne l'observons-nous pas sous la forme du désenchantement dont le domaine est encore trop souvent l'objet et que nous avons traduit par une " maladie de la communication interne " (Ely 2010) ? Dans un contexte interne de crise de confiance et de défiance, la soi-disant " communication interne » dont on fait largement référence - parfois non sans un certain angélisme -, se trouve bien souvent utopique, vide de son sens premier, réduite à une forme d'incommunication interne, galvaudée, dévoyée de sa finalité, substituée en simple information interne descendante, aveugle et pléthorique, en une transmission unilatérale et pyramidale de l'information, depuis l'organe directionnel, vers les salariés.

Peut-on nous satisfaire du sombre tableau que nous venons de brosser, dans ce chapitre de contextualisation, par cette vision d'apparence négative et pessimiste de la situation ? Sans réfuter pour autant notre constat, nous proposons maintenant une approche davantage antithétique de la question.

\section{Une confiance organisationnelle restaurée par une éthique des vertus : bases d'une utopie managériale?}

Selon Fukuyama (1997 : 151), la confiance apparaît dès lors qu'une communauté partage "un ensemble de valeurs morales qui laissent espérer des conduites régulières et honnêtes ». Qu'elle soit fondée sur la dissuasion, le calcul, la relation, la fiabilité, la prévisibilité, la justice, qu'elle soit cognitive ou affective..., qu'elle s'appuie sur une démarche éthique ou pas, la confiance organisationnelle (Neveu 2004) nous apparaît revêtir de multiples formes et de vertus. Par ailleurs, si la vertu peut être considérée comme une "valeur au cour du management d'une entreprise adaptée » (Prat dit Hauret \& Théron $2010: 163)$, toutes les conditions ne sont pas toujours réunies pour que confiance et vertu soient suffisamment effectives dans les pratiques organisationnelles, ainsi que peut le laisser supposer la première partie de notre travail. La recherche d'une restauration de la confiance organisationnelle par les vertus constituerait-elle alors une opportunité ou une utopie managériale, une réalité illusoire ou bien virtuelle? 


\section{Des vertus de la confiance organisationnelle}

$\mathrm{Au}$-delà de présenter le concept de confiance « dans tous ses états " (Simon 2007), ou encore de dresser une typologie des formes de confiance dans une pluralité de formes organisationnelles (Bornarel 2007), nous nous attacherons à rappeler ici certains apports de la confiance organisationnelle, en considérant sa position transversale, tant dans les relations interpersonnelles, en présentiel, que dans les relations au sein des groupes virtuels.

$\mathrm{Au}$ plan interpersonnel, si de nombreux travaux scientifiques existent en la matière, dont certains traitent des conditions d'une "dynamique de la confiance interpersonnelle (Karsenty $2011: 136$ ), nous avions spécifiquement approché par nous-même toute son importance dans une étude sur la relation assuré-assureur au point d'accueil de l'organisation observée (Ely 2007). Après une enquête qualitative auprès d'une population de personnels de contact, nous mettions en évidence que la relation commerciale, entre l'assuré et son assureur, pouvait être basée, de façon récurrente, sur des dimensions affectives, de confiance ou de rassurance. Cette relation nous est apparue telle que l'assureur (personnel de contact), dans ses rapports avec sa clientèle, faisait preuve d'une plus-value relationnelle de la plus haute importance, au même titre que, par analogie, l'infirmière participe, de par son savoir-être, son rôle propre, du traitement et de la guérison de son patient. Tout semble ainsi se passer comme si la relation de l'assuré à l'assurance positionnait ce dernier en situation de manque (d'information, de visibilité, d'assurance au plan psychologique, de compréhension...) telle qu'elle doit être psychologiquement compensée par une relation de confiance, construite avec le personnel de contact, au travers de la dimension relationnelle, humaine et non prescrite de son travail.

$\mathrm{Au}$ plan de la confiance dans les relations de groupe, cette dernière constitue d'autant le catalyseur du collectif, ou de la communauté, que ceux-ci s'inscrivent dans le cadre de conditions de travail virtuel ou de collaboration à distance. En considérant l'organisation virtuelle comme une organisation en réseau, émerge ainsi la notion d'entreprises virtuelles et leurs caractéristiques communes, telles que la dispersion géographique des partenaires, l'usage des TIC, l'innovation, et, surtout, un degré de confiance élevé, nécessaire pour pallier la faiblesse des contacts directs entre les individus (Coat \& Favier 2000), ce à quoi un certain «e-leadership» (Jawadi 2010 : 284) peut être associé comme facteur clé de performance.

Dans ces types de systèmes d'information collectifs, c'est, dans certaines conditions, la stratégie informationnelle de l'entreprise qui met tout en œuvre pour que l'information circule, le plus souvent par le biais d'un réseau interne d'entreprise, type réseau Intranet. La question de la confiance est cruciale dans le management de la connaissance qui est ici en œuvre : on suppose d'ailleurs l'existence d'une « confiance informationnelle reposant sur la perception des individus envers la qualité de l'information (et des sources d'information) et de sa valeur (symbolique, d'affaires, de preuve, etc.) » (Maurel ; Chebbi 2012 : 76). 
Dans le même ordre d'idée, la confiance organisationnelle traverse nécessairement toute forme d'intelligences : organisationnelle (Massé, Thibaut 2001), collective (Levy 2003 ; Zara 1997), économique (Bulinge 2002)... au point de constituer un véritable « capital social " (Fukuyama 1997 : 38) au sein de l'organisation.

\section{De la confiance envers les vertus organisationnelles}

Le mot «vertu » peut présenter un caractère moraliste voire démodé. En estil peut-être de même de tout rapprochement entre le monde du management et celui de la philosophie antique ? C'est pourtant, à nos yeux, bien le contraire, comme bien des auteurs ont pu le montrer (De Borchgrave 2006 ; Goy 2012). Nous pensons que l'universalité des vertus témoigne de toute leur légitimité dans l'actualité des problématiques managériales et organisationnelles. En quoi leur prise en compte pourrait-elle constituer un apport complémentaire aux pratiques managériales qui soit digne de confiance aux yeux du manager?

Des vertus des Anciens aux vertus spirituelles et sociales dans l'organisation

Pour les Anciens, la vertu - l'arétè des Grecs et la virtus des Latins -, c'est, en premier ressort, l'excellence d'une disposition ou d'une capacité à agir dans quelque domaine que ce soit. C'est ainsi cette puissance spécifique qui permet à l'Homme d'affirmer son Humanité : « Vertu, au sens général, c'est puissance ; et au sens particulier : humaine puissance ou puissance d'humanité. C'est ce qu'on appelle aussi les vertus morales, qui font qu'un homme semble plus humain ou plus excellent, comme disait Montaigne, qu'un autre, et sans lesquelles, comme disait Spinoza, nous serions à juste titre qualifiés d'inhumains » (Conte-Sponville 1995 : 9). De Socrate à Platon et Aristote et, bien après eux, les propagateurs d'une théorie de la vertu (Cicéron, Augustin, Boèce, Abélard...), sera laissé en héritage tout un patrimoine de valeurs et de réflexions dont nous mesurons toute la richesse lorsque nous tentons d'en établir quelques liens avec les réalités de la vie organisationnelle d'aujourd'hui : si la vertu est bien excellence, quelle serait donc cette excellence, ces vertus proprement humaines, susceptibles de (ré)générer confiance et lien social dans l'organisation ? N'y a-t-il pas quelques intérêts managériaux complémentaires, pour la performance de l'organisation, à transcender son cadre économique en prenant quelque hauteur de vue en référence à des vertus ou des valeurs telles que le respect de la "personne » (Mounier 1949), aussi humanistes qu'universelles, dans le sens d'une "spiritualisation de la vie économique " (Fukuyama $1997: 331$ ) ?

$\mathrm{Au}$-delà de tout angélisme et autres modes bien-pensantes, sept vertus proposées par Forasacco \& Voynnet-Fourboul (2010 : 74), en œuvre dans l'organisation, et fondées sur la spiritualité (qu'elles soient de l'ordre du Courage, de l'Intégrité, de la Tempérance, de la Sagesse, de la Justice, de la Gratitude, ou de la Compassion), pourraient ainsi constituer « une base 
d'action plus performante des organisations » (Ibid., p. 73). Ici, le business serait plutôt conçu via un "leadership spirituel " (Ibid.), au-delà de la production de résultats et de bénéfices, comme devant bénéficier à la société ou à la communauté.

Si un ensemble de vertus peut être convoqué dans l'organisation, selon un rapport strictement individuel et intime à une forme de spiritualitét, il est aussi une série de "vertus sociales, comme l'honnêteté, la fiabilité, l'esprit de coopération et un sens du devoir envers autrui, qui sont de nature foncièrement sociale " (Fukuyama $1997: 54)$. En tant que « réquisits du développement de vertus individuelles comme l'éthique du travail» (Ibid.: 57), les vertus sociales mettent en jeu un fort degré de confiance mutuelle, de solidarité sociale, et peuvent, en tant que " condition supplémentaire des relations économiques » (Ibid. : 149) augmenter l'efficacité économique.

\section{Management humaniste et éthique par les vertus morales}

Le management humaniste ${ }^{5}$ a pour principe et objectif « de protéger et favoriser le sentiment d'autodétermination de chaque salarié » (Arnaud 2008: 207). Selon la théorie de l'autodétermination, tous les êtres humains ont la volonté de s'autodéterminer. Dans cet esprit, favoriser, au sein des individus dans les groupes, leurs conditions d'autodétermination, leur motivation intrinsèque ${ }^{6}$, nous rapprocherait d'un management humaniste, si tant est que certains principes soient respectés : autonomie du salarié lui permettant d'exprimer sa personnalité et d'éprouver sa propre liberté ; développement des compétences (expression des talents et qualités, détermination de challenges à relever) ; reconnaissance du salarié à sa juste valeur et écoute, relations de confiance) ; lutte contre les discriminations (respect des libertés individuelles et collectives ) ; respect de la singularité et de la subjectivité de la personne (Arnaud $2008: 211-212$ ).

Dans le même ordre d'idée, au-delà d'un management éthique ${ }^{7}$, si l'on se réfère à une « dimension éthico-sociale de la compétence " (Arbouche 2008 : 121), en gestion des ressources humaines, les comportements éthiques sont

\footnotetext{
4 Compte tenu du caractère équivoque du thème de la spiritualité au travail, tant celle-ci est considérée comme relevant de l'intimité des salariés ou encore rattachée, par simplification logique, à la religion et autres formes de croyances en tous genres, nous retiendrons une définition plus universelle de la spiritualité, sélectionnée par Mériade \& Mainetti (2013: 126) comme « le sentiment fondamental de l'être relié à lui-même, aux autres et à l'univers tout entier» (Mitroff et Denton 1999 : 83).

5 « humaniste » dans le sens où ce qui est humaniste est « ce qui considère la personne comme une fin et jamais comme un moyen, ce qui favorise l'exercice de sa liberté, le développement de son autonomie morale, de son autodétermination, l'expression de ses talents, et des rencontres avec autrui permettant que s'établissent une reconnaissance et un respect mutuels. (Arnaud $2008: 211$ ).

6 Dans le cas de la motivation intrinsèque, l'activité est une fin en elle-même et non pas seulement le moyen d'atteindre d'autres fins. Se traduit par : " J'entreprends une tâche parce que je le veux et qu'elle me plaît » (Arnaud $2008: 216$ ).

7 Le management éthique, sur la base d'une éthique des organisations appliquée au management ou éthique managériale, opérationnalise cette dernière dans un certain nombre de techniques, de méthodes et de principes (Deslande 2012 : 4) dont par exemple la RSE (Responsabilité Sociétale des Entreprises) est une application.
} 
appréhendés sous l'angle du "savoir-être" qui suit le "savoir" et le "savoir-faire". Ce "savoir-être", appelle un postulat auquel nous nous référons : "l'éthique des vertus [...] est la plus appropriée aux pratiques de management en général et aux pratiques de gestion des ressources humaines en particulier» (Ibid. : 122), la vertu s'entendant ici comme « une qualité qui dispose en permanence à viser des fins bonnes [...] un habitus qui donne le pouvoir de bien agir " (Ibid. : 124).

Ceci étant posé, une organisation, sous le prisme des vertus morales, entendra l'intégration d'attitudes et de comportements profondément éthiques, cette " manière d'être persistante " (Ibid. : 125), associée, dans sa dimension personnelle, à « la modération, la discipline et la maitrise de soi [...] la justice dans les échanges, tant horizontaux que verticaux. " (Ibid.) Selon ce même auteur, le management de la compétence éthique par les vertus morales nécessite l'exercice de deux types de vertus : de l'intelligence pratique (essentiellement la prudence) afin d'orienter les activités vers une fin et ordonner, déterminer les bons moyens et celles de discipline personnelle (justice, courage et tempérance), pour harmoniser et rectifier les relations avec les autres, selon ce qui est droit (Ibid.: 125).

Reprenons maintenant notre questionnement : si la confiance organisationnelle s'avère aussi souhaitable que transversalement et virtuellement en présence dans l'organisation, entre les relations interpersonnelles, comme dans les dynamiques des groupes virtuels, le manager peut-il miser sur les vertus organisationnelles comme support d'enrichissement des pratiques du management des communautés et de la communication interne en particulier? Devant les faiblesses d'une confiance organisationnelle détériorée et une communication interne utopique, peut-il envisager de restaurer ces dernières en pariant sur les pratiques d'un management humaniste et éthique par les vertus, ou bien alors réduire ce dernier au titre d'une utopie managériale ?

En considérant que "Toute vertu recèle un vice caché. La confiance sans vigilance dégénère en laxisme " (Masson 2004 : 63), en tenant compte d'une nécessaire vigilance comme «le corollaire indispensable de la confiance. » (Ibid. : 64), peut-être alors que les vertus managériales - évoquées ci-dessus - d'intelligence pratique et de discipline personnelle devraient nous amener à répondre à nos interrogations en tentant de témoigner, respectivement, de prudence et de courage... C'est dans cet esprit que nous souhaitons traiter maintenant la poursuite de cette recherche, selon une approche plus pragmatique en lien plus étroit avec notre expérimentation.

\section{Vers une " maïeutique managériale de la confiance " dans l'organisation vertueuse : le cas du Groupware "Infocom"}

À l'inverse d'une défiance dont nous avons rappelé les effets contreproductifs, nous savons que la confiance organisationnelle, tout comme la motivation intrinsèque, peut apporter de multiples ressources (humaines) 
à l'organisation. Mais elle ne peut se décréter, ni se normaliser. Non sans s'armer d'optimisme, de courage et de prudence, le manager peut s'efforcer de la tisser dans les rapports entre les salariés au travail. Comment peut-il révéler cette confiance organisationnelle, virtuellement présente chez l'individu et dans les groupes?

Afin de rester en cohérence avec l'objet premier de cet article, nous allons tenter ici d'approcher une première réponse à cette question en nous concentrant d'avantage sur la dimension de «savoir être " - évoquée plus haut pour décrire les comportements éthiques - en jeu dans le cadre de notre rôle d'animateur ${ }^{8}$ du Groupware ${ }^{9}$ et du Forum "Infocom », situation expérimentale que nous avons mise en place, pendant trois années consécutives, au sein de l'organisation observée. Nous proposons de questionner deux vecteurs - complémentaires aux dimensions de "savoir » et "savoir faire ", détaillées par ailleurs (Ely 2011) -, qui nous semblent avoir joué un rôle essentiel dans la qualité du climat de confiance organisationnelle rencontré : l'exercice transversal d'une forme de management humaniste et éthique par les vertus morales, ajouté à ce que nous proposons d'appeler une maïeutique managériale de la confiance.

Groupware "Infocom " et management humaniste et éthique par les vertus morales

Nous avons cherché à faire émerger, au sein de l'équipe constituée, une certaine «vitalité » (Gramaccia 2001) du dispositif, en nous efforçant d'entretenir - quotidiennement et de multiples façons - un état d'esprit participatif, susceptible de favoriser une dynamique collective, faite de libre expression, de créativité, d'imagination, de responsabilité, de partage des savoirs et de confiance mutuelle. Sans l'installation d'un certain climat de confiance, indispensable à la construction de toute équipe, de surcroît, lorsqu'elle présente toutes les caractéristiques d'une équipe virtuelle (Gléonnec 2004), nous ne pouvions compter valoriser la participation au sein du groupe.

Pour aller plus loin, nous serions tenté de chercher à confronter la réalité du Groupware que nous avons piloté, à l'hypothèse d'un management humaniste et éthique par les vertus morales évoqué plus haut. Nous pourrions alors reprendre ici, par exemple, les différents principes énoncés de l'autodétermination, les deux types de vertus relevant de l'intelligence pratique (la prudence) ou de la discipline personnelle (justice, courage et tempérance), auxquelles nous associerions les sept vertus évoquées d'un leadership fondé sur la spiritualité (Forasacco, Voynnet-Fourboul, 2010 : 74). Sur ces bases, nous chercherions

8 Plus communément dénommé aujourd'hui « community manager» (Chauvin 2011).

9 Entendu comme "système d'information créé pour permettre aux membres d'un groupe de travailler ensemble électroniquement, grâce aux technologies de l'informatique et des télécoms, avec trois dimensions principales : les participants, les méthodes collectives de travail et les technologies. » (Coat \& Favier 2000). Le Groupware "Infocom » comprend in fine une équipe de 21 salariés participants, tous bénévoles, dans un ensemble représentatif des catégories socioprofessionnelles de l'organisation. 
volontiers à apprécier en quoi ces différentes variables et valeurs ont vu leur application - ou pas - dans la manière dont nous avons managé notre Groupware, pendant ses trois années successives d'exploitation. Nous passerions ainsi au crible notre «savoir être " managérial en la forme de nos attitudes et comportements humanistes, éthiques et vertueux...

Seulement, au plan méthodologique, sous le seul angle restreint de l'autoappréciation, notre démarche souffrirait d'un manque de distanciation, vraisemblablement amplifié par notre fort attachement personnel à ces dimensions éthiques et humanistes dont nous pensons, très subjectivement, nous en faire une règle de conduite personnelle. Si nous formulons l'hypothèse d'un rapport de causalité entre le bon fonctionnement et les résultats positifs du Groupware "Infocom ", son climat de confiance constructif, et un management humaniste et éthique par les vertus morales prétendument exercé par nos soins, il resterait alors à mettre en place un protocole méthodologique susceptible de nous permettre de le vérifier objectivement, en sollicitant l'opinion des 21 membres du dispositif via, par exemple, une démarche d'enquêtes qualitatives...

Groupware «Infocom » et " maïeutique managériale de la confiance »

Afin de favoriser la création et l'entretien du sens, de maintenir un certain niveau d'implication, de motivation et de valorisation personnelles et collectives chez les participants du groupe, de répartir et de synchroniser au mieux les multiples ressources internes, de favoriser l'intelligence collective entre les participants, nous avons tenu, au travers de notre rôle d'animateur, à adopter un style de management évitant toute forme de dirigisme de notre part. Nous avons veillé, en cela, à faire tout notre possible pour favoriser le respect de la personne et la révélation de ses ressources créatives, trop peu souvent sollicitées - voire bafouées - par un management directif dont elle dépend au sein de la pyramide technocratique.

Dans ce sens, nous nous sommes aperçu que notre mode de pilotage du Groupware, en phase, selon nous, avec les formes de management humaniste et éthique évoqué plus haut, avait la particularité de favoriser systématiquement, dans les pratiques, l'autodétermination de la personne pour stimuler, le plus possible, sa motivation intrinsèque, autorégulée. Fortement inspiré d'une approche par l' "écoute en profondeur " (Crozier 1997), dans l'esprit du " management d'une écoute interne de l'organisation "(Ely 2011), en cohérence avec notre posture hiérarchique quotidiennement «en retrait » (en rupture avec les pratiques managériales hyperdirectives de l'organisation observée), nous avons fait d'emblée adhérer toute l'équipe à un principe de fonctionnement fort, une sorte de leitmotiv affirmant qu' "Il n'y a pas de communication sans écoute et pas d'écoute sans système d'information ». Nous avons régulièrement sollicité les membres du Groupware, en valorisant leurs capacités individuelles - mais aussi collectives - à s'inscrire dans une démarche active de 
participation, de contribution au projet collectif, via notre média intranet principal, le Forum "Infocom ». Au-delà de l'influence d'un " e-leadership » - qu'il resterait à démontrer -, suite à nos sollicitations individuelles et collectives, suite à notre animation, à notre coaching de Community manager (Chauvin 2011 ; Chéreau 2010), en la forme de multiples questions posées à chaque membre ou à la communauté entière, s'est installé progressivement un intense jeu de questions-réponses postées sur le forum (plus de 2000 messages pertinents en trois années d'exploitation (Ely 2011 : 262). Comme véritable clé de voûte reconnue de ce type de dispositif numérique auquel chaque participant avait accès, les questions-réponses ont ainsi généré une intensité de communication interpersonnelle et une dynamique communicationnelle interne et participative (Ely 2005b : 18).

Aussi, au même titre que nous faisions référence aux richesses de la pensée antique, ces premiers éléments de notre questionnement sur nos pratiques managériales, nous apparaissent faire l'écho, dans une certaine mesure, d'une forme d'approche discursive par l'Art de la maïeutique socratique. En effet, si la pensée socratique peut s'avérer celle d'un " philosophe au secours de l'entreprise "(Goy 2012), nous constatons, dans nos pratiques, plusieurs convergences opératoires avec la maïeutique, cet « art d'accoucher » les esprits d'une solution, ce procédé utilisé par Socrate et rapporté par Platon dans le Théétète (Chambry 1967). Rappelons que la mère de Socrate étant sagefemme, celui-ci exerçait sur les âmes cet Art de la maïeutique que sa mère avait exercé sur les femmes. Socrate n'enfantant rien, se disant lui-même stérile, se connaissant par son célèbre "Connais-toi toi même ", prétendait qu'il savait qu'il ne savait rien. Il se plaçait ainsi comme l'instrument qui vient en aide aux autres âmes en forçant les hommes à révéler, à accoucher, de ce dont ils étaient porteurs, quelquefois sans le savoir. Faire ressurgir ainsi des connaissances oubliées, par la stimulation d'une co-construction dialectique, cet Art de la Maïeutique, en la forme de techniques empathiques de questionnement ou de médiation, retient toute notre attention, tant nos pratiques semblent présenter certaines convergences intéressantes, à commencer par ce dialogue intense que nous avons favorisé via le Forum "Infocom ». Ce jeu d'alternances de questions-réponses dont nous avons favorisé la pratique, aura permis de mettre en place, progressivement, avec les personnes, un dialogue intense, bien qu'à distance, et à l' "accoucheur" que nous pensons avoir été, de constater des résultats collectifs générant, dans le groupe, cinq grands invariants : motivation, participation, co-construction, communication interpersonnelle et résolution de problèmes (Ely 2005b : 189). C'est principalement autour de ce dialogue-là, incitant les membres du dispositif à rechercher, dans et par eux-mêmes, des ressources qu'ils possèdent en eux-mêmes, ainsi qu'autour de la confiance solidement installée entre ces derniers, ajoutée à notre attitude d'écoute en profondeur, que nous sommes amené à constater une certaine convergence entre notre mode de pilotage 
et les apports de la maïeutique socratique. À cette apparente conjonction, s'ajoutent celle représentée par nos appuis sur notre posture hiérarchique en retrait, nos systématiques sollicitations et valorisations sincères des personnes de notre équipe et de leur motivation intrinsèque, et notre mode d'animation d'une forme d'autodétermination des participants.

\section{Conclusion}

Nous nous sommes proposé comme objectif, au cours de cette recherche exploratoire, de questionner la confiance organisationnelle devant un environnement de menaces et d'opportunités. Pour les premières, nous avons rappelé plusieurs facteurs sociétaux de détérioration de la confiance, en une défiance contre-productive pour l'organisation, ce qui nous a permis de proposer le constat d'une utopie de la communication interne, constat d'autant plus problématique que nous avons retenu la communication interne comme " outil du management" (Mucchielli 2001).

Pour les secondes, nous nous sommes demandé si un rapprochement original entre le monde du management et celui de la philosophie, via un management humaniste et éthique par les vertus morales, associé aux apports de la maïeutique socratique, pouvait constituer une opportunité - ou une utopie managériale - dans la recherche d'une restauration de la confiance organisationnelle.

Comme début de réponse à notre questionnement, en nous appuyant sur l'observation de notre rôle d'animateur du Groupware et du Forum "Infocom» que nous avons exercé durant trois années successives, dans le cadre de notre expérimentation, plusieurs confluences d'approche - notamment autour de l'instauration du dialogue, de la confiance et de l'écoute - nous permettent de poser l'hypothèse d'une convergence entre nos pratiques de pilotage du dispositif et la maïeutique socratique, en la forme d'une démarche que nous proposons d'appeler maïentique managériale de la confiance.

Si Socrate prétendait avoir "l'Art d'accoucher les esprits ", de libérer les âmes des individus, une certaine maïeutique managériale de la confiance, appliquée au pilotage de notre dispositif, semble être à même de favoriser, d'enfanter, d'une certaine âme du collectif, cet esprit communautaire que le Community Manager est censé construire, en phase avec la définition de la communication interne que nous avions initialement retenue comme « ensemble de principes d'actions et de pratiques visant à donner du sens et favoriser l'appropriation, à donner de l'âme pour favoriser la cohésion et inciter chacun à mieux communiquer pour favoriser le travail en commun " (Détrie \& Broyez 2001). Les résultats positifs, au plan qualitatif et quantitatif de notre dispositif, nous confortent dans ce sens, et nous pousseraient ainsi à considérer que cet enfantement des âmes individuelles est à la maïeutique socratique ce que la maïentique 
managériale de la confiance serait à l'âme du collectif, à l'esprit communautaire d'un Groupware ou d'une communauté virtuelle.

Au-delà de tout prosélytisme à l'égard d'une proposition pouvant constituer la base d'un questionnement sur un nouveau paradigme de la communication interne, nous observons certaines limites théoriques et méthodologiques qui nécessiteraient un investissement complémentaire, à commencer par tout un protocole scientifique de validation d'hypothèses que nous voudrions mettre en œuvre ultérieurement comme seconde étape de cette recherche.

Néanmoins, devant un contexte interne et sociétal de crise du management, de perte de sens, de défiance, de désacralisation et de déshumanisation du travail, nous pensons qu'un approfondissement et une application de cette proposition pourraient offrir de réelles perspectives de recherche scientifique, dans les trois domaines-phares - et liés - de la communication d'entreprise, des RH, et du management (dont celui de la connaissance), susceptibles de favoriser, sous certaines conditions, un certain management humaniste et éthique par les vertus morales, porteurs d'humanité et de spiritualité encore trop souvent méprisées dans l'organisation.

\section{BIBLIOGRAPHIE}

ALGAN Y. \& CAHUC P. (2007), La société de défiance. Comment le modèle social français s'autodétruit, Paris, Rue d'ULM.

ALGAN Y., CAHUC P. \& ZYLBERBERG A. (2012), La fabrique de la défiance.... et comment s'en sortir, Paris, Albin Michel.

ARBOUCHE M. (2008), Développement des compétences éthiques. Une approche par l'éthique des vertus, Management $\mathcal{E}^{\circ}$ Avenir, n 20, p. 115-128,

ARNAUD S. (2008), Le management humaniste, Revue internationale de psychosociologie n ${ }^{\circ}$ 34, p. 207-224.

AURIAC J. \& CHANON A. (2012), L'entreprise à l'ère de la défiance : de l'intérêt du dialogue sociétal,

http://www.sudoc.abes.fr/DB=2.1/SET=2/TTL=1/CLK?IKT=12\&TRM=162340974 Paris, L'Harmattan.

BATAZZI C. (2005), L'éthique dans la communication interne des dirigeants : entre comportement opportuniste et projet humaniste, in La communication des organisations $\grave{a}$ la croisée des chemins disciplinaires, Grec/o, Paris, L'Harmattan.

BORNAREL F. (2007), La confiance comme instrument d'analyse de l'organisation, Revue française de gestion, $\mathrm{n}^{\circ} 175$, p. 95.

BRETON P. (1997), L'utopie de la communication : le mythe du village planétaire, Paris, La Découverte.

BULINGE F. (2002), Pour une culture de l'information dans les petites et moyennes organisations : un modèle incrémental d'Intelligence économique, Thèse en sciences de l'information et de la communication, Université du Sud Toulon Var.

CHAMBRY E. (1967), Platon Théétète, Parménide, Paris, Flammarion, [En ligne, consulté le 01/10/14], http://gallica.bnf.fr/ark:/12148/bpt6k3797x/f4.image 
CHAMPEAUX J. \& BRET C. (2000), La Cyber Entreprise, Paris, Dunod.

CHAUVIN P. (2011), Le Community Management: nouvelle approche de la communication, Paris, Gualino-Lextenso.

CHÉREAU M., (2010), Community management, Paris, Dunod.

CLOT Y. (2010), Le travail à cceur, pour en finir avec les risques psychosociaux, La Découverte, Paris.

COAT F. \& FAVIER M. (2000), La réalité de la virtualité: Le cas des équipes virtuelles, 5 Colloque de l'AIM, 8-10 novembre, Montpellier, France.

COMTE-SPONVILLE A. (1995), Petit traité des grandes vertus, Paris, Point deux.

CROZIER M. (1997), L'Entreprise à l'écoute, Point, La Flèche.

CROZIER M. \& FRIEDBERG E. (1977), L'acteur et le système, Paris, Seuil.

DE BORCHGRAVE R. (2006), Le philosophe et le manager : penser autrement le management, Bruxelles, De Bøck Supérieur.

DECI, E \& RYAN, R. (2000), The What and Why of Goal Pursuits : Human Needs and the Self-Determination of Behavior, Psychological Inquiry, p. 227-268.

DEJOURS C. (1998), Souffrance en France, Seuil, Paris.

DEJOURS C. (2000), Travail, usure mentale, Bayard, Paris.

DEJOURS C. \& BËGUE F. (2009), Suicide au travail : que faire? Paris, PUF.

DES ISNARD A. \& ZUBER T. (2008), L'open space m’a tuer, Hachette littérature, Paris.

DESLANDES G. (2010), Ethique des organisations : le retour de la vertu, L'Expansion Management Revierw, n ${ }^{\circ} 137$, L'Express, Paris, Roularta.

DESLANDES G. (2012), Le management Ethique, Management Sup, Paris, Dunod.

DÉTRIE P. \& BROYEZ C. (2001), La communication interne au service du management, Paris, Liaisons.

DIEGUEZ S. (2009), Le syndrome d'Hubris, la maladie du pouvoir. Cerveau $\varepsilon^{\circ}$ Psycho, n 34, juillet-août 2009, pp. 24-30.

DUFOUR D-R. (2003), L'art de réduire les têtes. Sur la nouvelle servitude de l'homme libéré à l'ère du capitalisme total, Paris, Denoël.

DUTERME C. (2002), La communication interne en entreprise. L'approche de Palo Alto et l'analyse des organisations, De Boeck Université, Bruxelles.

ELY F. (2005a), DIRCOM \& TIC : le DirCMO, quand l'anagramme préfigure la révolution de la fonction...vers l' e-dircom, $I s d m \mathrm{n}^{\circ} 21$, article $\mathrm{n}^{\circ} 252$, [consulté en ligne le 01/10/14], http://isdm.univ-tln.fr/articles/num_archives.htm\#isdm21

ELY F. (2005b), Le forum Intranet de discussion : un nouveau dispositif stratégique de communication interne des organisations ?, Communication \& Organisation $\mathrm{n}^{\circ}$ 28, $2^{\mathrm{e}}$ semestre 2005, Bordeaux 3, Greco [consulté en ligne le 01/10/14], http:// communicationorganisation.revues.org/3350?lang $=\mathrm{fr}$

ELY F. (2007), Dircom et Technologies de l'Information, de la Communication et de la Collaboration : le e-Dircom, pour une Intelligence Communicationnelle Interne de l'organisation, Thèse en Sciences de l'Information et de la Communication, Université du Sud Toulon Var, [consulté en ligne le 01/10/14], http://archivesic.ccsd.cnrs.fr/index. 
php?action_todo=search\&view_this_doc=sic_00349806\&version=1\&halsid=jdt6lumkc hml326otgg3r1nom0

ELY F. (2010), Un schisme entre management et communication : pour une intelligence communicationnelle interne de l'organisation. In Management et communication : Mutations, emprunts et résonances, Paris, L'Harmattan. [consulté en ligne le 01/10/14], http://www.editions-harmattan.fr/index.asp?navig=catalogue\&obj=livre\&no=32952

ELY F. (2011), Les opportunités du numérique, pour une réhabilitation de la fonction communication comme levier du management de l'organisation, in Management et prospective $\mathrm{n}^{\circ} 1$, Cormelles-le-Royal, et colloque La communication numérique demain : quels métiers, quelles compétences, quels managements, quelles organisations du travail et quelles formations ?, le 18 mai 2011, ESSEC, Paris La Défense.

FOULARD C. (1998), L'entreprise communicante, Paris, Hermès.

FORASACCO C. \& VOYNNET-FOURBOUL C. (2010), Les vertus du leadership fondé sur la spiritualité, in Tous vertueux, sous la direction de Jean-Marie Peretti, Eyrolles, Paris, Éd. d'Organisation.

FUKUYAMA F. (1997), La confiance et la puissance : vertus sociales et prospérité économique, Paris, Plon.

GLÉONNEC M. (2004), Travailler ensemble à distance: une question de confiance, Hermès, La Revue, n 39, p. 18-26, [consulté en ligne le 01/10/14], www.cairn.info/revuehermes-la-revue-2004-2-page-18.htm

GORZ A. (1988), Métamorphoses du travail. Quête du sens, Paris, Galilée.

GOY D. (2012), Socrate: un philosophe au secours de l'entreprise, Paris, Maxima.

GRAMACCIA G. (2001), Les actes de langage dans les organisations, Paris, L'Harmattan.

ELLUL J. (1977), Le système technicien, Paris, Calmann-Levy.

HADDAD G. (2013), Tripalium, Pourquoi le travail est devenu souffrance, Paris, François Bourin Éditeur.

HUISMAN D. (1985), L’incommunication: essai sur quelques effets pléthoriques abusifs ou pervers de la communication actuelle, Paris, Vrin.

HIRIGOYEN M. F. (2003), Le harcèlement moral, la violence perverse au quotidien. Paris, La Découverte.

HOPQUIN B. (2008), Cadres. La Comédie du Bonheur, in Le Monde, 18 septembre 2008.

JAWADI N. (2010), Leadership et gestion de la confiance et de la performance dans les équipes virtuelles : une approche par la complexité comportementale, Management $\mathcal{E}^{\circ}$ Avenir n ${ }^{\circ}$ 37, p. 282-305.

KARSENTY L. (2011), Confiance interpersonnelle et communications de travail. Le cas de la relève de poste, in Le travail humain, Paris, PUF.

LÉVY P. (2003), Le jeu de l'intelligence collective, Sociétés n 79, p. 105-122, [consulté en ligne le 01/10/14], www.cairn.info/revue-societes-2003-1-page-105.htm.

LEVY P. (1997), L'Intelligence collective. Pour une anthropologie du cyberespace, Paris, La Découverte.

LOJKINE J. \& MALETRAS J-L. (2003), Le temps de travail informationnel : des mesures incertaines et contradictoires, Revue de l'Ires $\mathrm{n}^{\circ} 42$. 
MASSÉ G. \& THIBAUT F. (2001), Intelligence économique, un guide pour une économie de l'intelligence, Bruxelles, De Boeck Université.

MASSON P. (2004), Manager bumaniste, Paris, Éditions d'Organisation.

MAUREL D. \& CHEBBI A. (2012), La perception de la confiance informationnelle, Communication et organisation, $n^{\circ} 42$, p. 73-90, Bordeaux, Presses Universitaires de Bordeaux.

MEDA D., (1996). Le travail, une valeur en voie de disparition? Paris, Aubier.

MÉRIADE L. \& MAINETTI N. (2013), La spiritualité : Quelles places pour la spiritualité dans les entreprises ? L'exemple chinois, Revue Internationale de Psychosociologie et de Gestion des Comportements Organisationnels n ${ }^{\circ}$ 48, ESKA, p. 125-144.

MINTZBERG H. (2004), Le management, voyage au centre des organisations, Éditions d'organisation, Paris.

MITROFF I. \& DENTON E. (1999), A Spiritual Audit of Corporate America. JosseyBass, San Francisco.

MOUNIER E. (1949), Le personnalisme, Coll. Que sais-je ?, Paris, PUF.

MUCCHIELLI A. (1999), Théorie systémique des communications, Paris, Armand Colin.

MUCCHIELLI A. (2001), La communication interne, Paris, Armand Colin.

MUCCHIELLI A. (2004), Le développement des méthodes qualitatives et l'approche constructiviste des phénomènes humains, in Recherche qualitative, Hors-Série, Numéro 1, actes du Colloque Recherche qualitative et production de savoirs, Uqam, 12 mai 2004.

NEVEU V. (2004), La confiance organisationnelle: définition et mesure, Actes du XV ${ }^{\mathrm{e}}$ Congrès de l'AGRH, Montréal.

PEZÉ M. (2010), Ils ne mouraient pas tous mais tous étaient frappés, Flammarion, Paris.

PETER L. \& HULL R. (1970), Le principe de Peter, Paris, Stock.

PRAT DIT HAURET C. \& THÉRON O. (2010), La vertu, une valeur au cœur du management d'une entreprise adaptée, in Tous vertueux, sous la direction de Jean-Marie Peretti, Paris, Eyrolles, Éd. d'Organisation.

RIFKIN J. (2006), La fin du travail, La découverte, Paris.

SALENGRO B. (2005), Le stress des cadres, Paris, L'Harmattan,

SFEZ L.(1988), Critique de la communication, Paris, Seuil,

SIMON E. (2007), La confiance dans tous ses états, Revue française de gestion $\mathrm{n}^{\circ} 175$, p. 83-94

TNS Sofres (2009), Salariés et sortie de crise, octobre 2009, [consulté en ligne le 01/10/14], http://www.tns-sofres.com/etudes-et-points-de-vue/salaries-et-sortie-de-crise\#

WINKIN Y. (1996), Anthropologie de la Communication, Paris, Essais.

ZARA O.(2005), Le management de l'Intelligence collective. Vers une nouvelle gouvernance, Paris, M2 Éditions.

Résumé : La présente recherche exploratoire, dans le cadre des rapports complexes entre communication des organisations et management, questionne le concept de confiance organisationnelle, face à certaines menaces socioprofessionnelles, en la forme 
d'une défiance contre-productive pour l'organisation et d'une utopie de la communication interne, mais aussi devant le champ - encore peu exploré -, des opportunités d'un rapprochement original entre management, philosophie, vertus morales et maïeutique socratique. Sur la base des résultats obtenus à la suite d'un travail de thèse, notre terrain est un dispositif numérique de collaboration et de communication interne que nous avons piloté, durant trois années successives, au sein d'une grande entreprise française d'assurance. En y examinant nos pratiques de Community Manager, nous approchons un mode de pilotage que nous nous sommes efforcé d'appliquer en la forme originale d'une maïeutique managériale de la confiance.

Mots-clés: Communication interne utopique, Maïeutique managériale, Confiance organisationnelle, Management humaniste et éthique, Vertus organisationnelles, Numérique.

Abstract: This exploratory research, within the context of the complex relationships between communication of organizations and management, questions the concept of organizational trust, in front of social and professionnal threats, in the shape of counterproductive mistrust for the organization and utopia of internal communication, but also in front of a field that hasn't been much explored yet: the opportunities of an original link between management, philosophy, moral virtues and Socratic maieutics. Based on thesis results, our ground is a digital device of collaboration and internal communication which we managed, during three successive years, within a french large company of insurance. By examining our practices of Community Manager, we approach a mode of management which we tried to apply in the original shape of managerial maieutics of trust.

Keywords: Utopian internal communication, Managerial Maieutics, Organizational trust, Humanist and ethical management, Organizational virtues, Digital technology. 
